\title{
Research Article Flexible Bus Route Optimization Scheduling Model
}

\author{
Ji-yang Sun, ${ }^{1}$ Yan-yan Chen $\mathbb{D},{ }^{2}$ Jian-ling Huang, ${ }^{3}$ Pan-yi Wei $\mathbb{D},{ }^{1}$ and Cheng-cheng Song ${ }^{1}$ \\ ${ }^{1}$ Beijing Key Laboratory of Traffic Engineering, Beijing University of Technology, Beijing 100124, China \\ ${ }^{2}$ Center of Cooperative Innovation for Beijing Metropolitan Transportation, Beijing 100124, China \\ ${ }^{3}$ Beijing Transportation Information Center, Beijing 100161, China \\ Correspondence should be addressed to Pan-yi Wei; weipanyi@foxmail.com
}

Received 28 June 2020; Revised 7 December 2020; Accepted 28 January 2021; Published 27 February 2021

Academic Editor: Shazim Memon

Copyright (c) 2021 Ji-yang Sun et al. This is an open access article distributed under the Creative Commons Attribution License, which permits unrestricted use, distribution, and reproduction in any medium, provided the original work is properly cited.

\begin{abstract}
A flexible bus route optimization scheduling model that considers the dynamic changes of passenger demand is proposed to address the large difference in demand for flexible bus passengers and real-time variability. This model uses the heuristic algorithm based on gravity model to determine the following: passenger booking; vehicle passenger capacity; team known conditions such as size, according to the dynamic changes of passenger demand for real-time iterative update shuttle travel time; vehicle operating costs (vehicle); and time cost for passengers (passengers waiting time for the vehicle, actual time of arrival, and the difference between expected and actual times of arrival) before minimization as the target. Finally, the practicabilities of the model and algorithm are verified by an example. Analysis results show that for 102 travel demands of 15 randomly generated demand points, completing all services requires 17-21 vehicles with average travel time of 24.59 minutes each. The solution time of 100 groups of data is within 25 seconds and the average calculation time is 12.04 seconds. Under the premise of real-time adjustment of connection planning time, this optimization model can thus better meet the dynamic demand of passengers compared with the current scenario. The model effectively reduces the planning path error, shortens the travel distance and passenger travel time, and achieves better results than the flexible bus scheduling model that ignores changes of connection travel time.
\end{abstract}

\section{Introduction}

How to realize optimal scheduling is the main problem faced by urban public transport operation and management. A good bus scheduling system can quickly optimize and adjust the line operation plan according to the travel needs of passengers. In addition, this system can improve the line service rate, reduce running time, and reduce travel time cost of passengers [1-3]. The traditional bus route optimization method is mainly designed based on experience, long-term observation, or IC card data statistical analysis. The objective is to extend, shorten, add, or delete certain lines, adjusting to the optimization and priority that meet the passenger demand of large passenger flow site. This method is mainly suitable for fixed-line bus route optimization with long cycle adjustment [4-7]. The emergence of flexible buses provides the possibility for dynamic optimization and adjustment of routes [7-10]. Vincent et al. [11] proposed a mixed-integer linear programming model and a variable neighborhood search to deal with the flexible vehicle and crew scheduling problem, by solving the problem that urban bus transport agencies have to assign their resources (vehicles and drivers) to cover timetables generated at the tactical level. Giorgio et al. [12] explored the potential contribution that the public transport agency can make to the emerging mobility as a service (MaaS) paradigm through the integration of regular collective transport services with complementary flexible transport schemes and other forms of shared-use transport. Nourbakhsh and Ouyang [13] presented an alternative flexible-route transit system in which each bus is allowed to travel across a predetermined area to serve passengers, analyzed the agency and user cost components of this proposed system in idealized square cities, and sought the optimum network layout, service area of each bus, and bus 
headway, to minimize the total system cost. Mulley and John [14] presented a viewpoint through their research that wellimplemented FTS has the potential to revitalise bus-based public transport services which are traditionally based on fixed networks with variable geographical coverage and levels of service. Jenny et al. [15] designed a multicommodity network flow programming model and obtained exact routing solutions using Lagrangian relaxation and a dynamic programming algorithm. Quadrifoglio and Dessouky [16] established the mixed-integer programming equation of system route optimization through analysis of key parameters of the flexible bus system. They likewise conducted simulation verification analysis on the system route design and scheduling problems to correct the model parameters [17]. Fu et al. [18] used super network to simultaneously simulate user activities and travel behaviors and then established a bus route selection model according to user travel behavior characteristics. Koffman [19] proposed an intelligent scheduling algorithm for urban public transport based on multiobjective demands. Tsubouchi [20] used the minimum spanning tree to find the optimal bus path. Xiong [21] developed an optimization method of community bussubway connection route considering potential demand. Qian-fei et al. [22, 23] established a hyperpath bus transport path adjustment model by deducing the expected waiting time of passengers and the probable number of passengers on the route. Pan [24] proposed a flexible bus route optimization and scheduling method based on long reservation. Guo [25] offered a customized bus route optimization selection method based on demand response. These studies are all flexible bus route optimization algorithms established according to passengers' reservation needs. However, the preconditions are that passengers make advance appointments or assume that passenger needs are known, that is, the methods are all based on "static demands." However, travel often requires short or instant reservation according to passenger needs. Flexible buses need to calculate the change of vehicle connection travel time caused by changes according to the "dynamic demand" of passengers. And these buses need to adjust routes to achieve dynamic optimization. In addition, the flexible bus connection modes in almost all the research studies mentioned above are in form of "many-to-one," that is, multiple flexible stations corresponding to one target station. The case of multiple flexible sites corresponding to multiple target sites (the "many-tomany" pattern) has rarely been studied.

With such consideration, the present study proposes a flexible bus route optimization scheduling method based on passenger dynamic demand and the "many-to-many" pattern. This method considers dynamic changes of passenger demand and the resulting changes of vehicle connection travel time. A flexible bus route optimization scheduling model is constructed on the basis of the known passenger carrying capacity and vehicle fleet size. Then, the connection trip time is updated in real time according to the dynamic variation characteristics of passenger demand and considering the time cost of vehicle operation and passenger travel.

\section{Problem Description and Modeling}

2.1. Problem Description. A flexible bus is a demand-based transportation system that can gather individual passengers' travel needs and provide personalized transportation services. Under the "dynamic real-time" demand mode, which is the focus of this study, the flexible bus system has high requirements on algorithm and operation cost control. The following assumptions, parameter selection, and modeling are carried out to establish a reasonable and appropriate model. Several factors such as passenger demand and operating cost are likewise considered.

2.2. Model Assumptions. The following assumptions are proposed, with (1) and (2) as dynamic hypotheses and (3)-(6) as static hypotheses:

(1) Passenger reservation demand changes dynamically at each station.

(2) The travel time between stations is dynamic.

(3) Location of each site is known.

(4) After booking, the time for each passenger to arrive at the target station is known.

(5) The service time for passengers to board the train is constant.

(6) The passenger-carrying capacity of the transfer vehicle is known.

2.3. Model Parameters. Table 1 shows the definition and description of the input and decision variables in the model.

2.4. Model Presentation. The coordination scheduling optimization model can be expressed as the following nonlinear programming model.

$$
\min \left[\sum_{i \in H \cup D} \sum_{j \in H \cup D} \sum_{k \in K} c_{i j} Y_{i j k}+\sum_{r \in R} a_{r}+\sum_{r \in R} \sum_{k \in K} \sum_{t}\left(X_{r k t} T_{t}-e_{r}\right)\right] .
$$

Equation (1) is the objective equation, which includes the minimization of the sum of three factors: (1) travel time of all vehicles to reduce operating costs; (2) sum of waiting time of all passengers in each station; and (3) sum of waiting time of all passengers in the target station. The latter two are determined to reduce the passenger time cost.

$$
\begin{gathered}
\sum_{j \in H \cup D} \sum_{k \in K} Y_{i j k} \geq 1, \quad \forall i \in H, \\
\sum_{j \in H \cup D} \sum_{k \in K} Y_{i j k} \leq V, \quad \forall i \in H .
\end{gathered}
$$

Constraints (2) and (3) indicate that for each demand point, at least one vehicle service and at most $V$ vehicle service are available. 
TABLE 1: Model parameter.

\begin{tabular}{|c|c|c|}
\hline Variable & Definitions and descriptions & Parameter type \\
\hline$H$ & Passenger demand station set & Set of integers \\
\hline$D$ & Target station set & Set of integers \\
\hline$K$ & Shuttle operation vehicle set & Set of integers \\
\hline$R$ & Passenger demand set & Set of integers \\
\hline$N$ & Total passenger demand, $N \geq 0$ & Parameters \\
\hline$V$ & Shuttle fleet size, $V \geq 0$ & Parameters \\
\hline$M$ & A large constant & Constant \\
\hline$T_{t}$ & The $t$ th departure time of the target station & $\begin{array}{l}\text { Decision } \\
\text { variables }\end{array}$ \\
\hline$d_{r}$ & Target stations $d$ of passenger demands $r$ & $\begin{array}{l}\text { Decision } \\
\text { variables }\end{array}$ \\
\hline $\operatorname{pr}$ & Pickup station $p$ for passenger demand $r$ & $\begin{array}{l}\text { Decision } \\
\text { variables }\end{array}$ \\
\hline$T_{r}$ & The starting time of the target station for passenger demand $r$ & $\begin{array}{l}\text { Decision } \\
\text { variables }\end{array}$ \\
\hline$c_{i j}$ & The travelling time between station $i$ and station $j$ & $\begin{array}{l}\text { Decision } \\
\text { variables }\end{array}$ \\
\hline$Q_{k}$ & The capacity of shuttle bus $k, Q_{K} \geq 0$ & $\begin{array}{l}\text { Decision } \\
\text { variables }\end{array}$ \\
\hline$X_{r k t}$ & If shuttle bus $k$ connects demand $r$ to the target station at time $t, X_{r k t}=1$; otherwise, $X_{r k t}=0$ & $\begin{array}{l}\text { Decision } \\
\text { variables }\end{array}$ \\
\hline$Y_{i j k}$ & If shuttle bus $k$ selects the $\operatorname{link}(i, j)$ as driving path, $Y_{i j k}=1$; otherwise, $Y_{i j k}=0$ & $\begin{array}{l}\text { Decision } \\
\text { variables }\end{array}$ \\
\hline$a_{r}$ & The time when the shuttle bus reaches the demand point where the demand $r$ is located & $\begin{array}{l}\text { Decision } \\
\text { variables }\end{array}$ \\
\hline$a_{s}$ & Travel time of the shuttle bus to reach the required point & $\begin{array}{l}\text { Decision } \\
\text { variables }\end{array}$ \\
\hline$e_{r}$ & Time of passenger demand rreaches target station & $\begin{array}{l}\text { Decision } \\
\text { variables }\end{array}$ \\
\hline$U_{i k}$ & $\begin{array}{l}\text { Auxiliary variable is used to ensure that there is no loop in the driving path of the connecting vehicle } K ; i \\
\text { indicates the shuttle bus passing through the station; if shuttle bus } k \text { leaves station } i, U_{i k}=0 \text {; if shuttle bus } k \\
\text { arrives station } i, U_{i k}=1\end{array}$ & $\begin{array}{l}\text { Auxiliary } \\
\text { variable }\end{array}$ \\
\hline
\end{tabular}

$$
\sum_{i \in H} \sum_{k \in K} Y_{i j k} \leq V, \quad \forall j \in D
$$

Constraint (4) means that at most $V$ vehicles participate in the operation.

$$
\sum_{j \in H \cup D} Y_{i j k}-\sum_{p \in H} Y_{p i k} \geq 0, \quad \forall i \in H, k \in K .
$$

Constraint (5) indicates that in addition to the first demand point of each vehicle service, the number of vehicles entering should be equal to that leaving to serve the other demand points.

$$
U_{i k}-U_{j k}+|H| \times Y_{i j k} \leq|H|-1, \quad \forall i, j \in H \cup D, k \in K .
$$

Constraint (6) avoids loops in the vehicle path.

$$
\begin{aligned}
& \sum_{i \in H} \sum_{j \in D} Y_{i j k} \geq 1, \quad \forall k \in K, \\
& \sum_{i \in H} \sum_{j \in D} Y_{i j k} \leq 0, \quad \forall k \in K .
\end{aligned}
$$

Constraints (7) and (8) ensure that each vehicle delivers passengers to their final destination.

$$
\sum_{r \in R} \sum_{t \in T} X_{r k t} \leq Q_{k}, \quad \forall k \in K
$$

Constraint (9) ensures that the capacity of each feeder vehicle does not exceed its rated capacity to ensure the service level.

$$
\sum_{k \in K} \sum_{t \in T} X_{r k t}=1, \quad \forall r \in R
$$

Constraint (10) means that any requirement can only be served by one vehicle.

$$
\sum_{m \in T /\{t\}} X_{s k m}-\left(1-X_{r k t}\right) M \leq 0, \quad \forall r, s \in R, \forall k \in K, \forall t \in T .
$$

Constraint (11) indicates that a transfer vehicle can only connect passengers at one departure time of a target station.

$$
\sum_{r \in R} \sum_{k \in K} \sum_{t \in T} X_{r k t}=N
$$

Constraint (12) guarantees that all demand served is equal to the known reservation demand. 


$$
\begin{aligned}
& a_{r}+c_{p_{r} p_{s}}-a_{s}+Y_{p_{r} p_{s} k} M \leq M, \quad \forall r, s \in R, \forall k \in K, \\
& a_{s}-a_{r}-c_{p_{r} p_{s}}+Y_{p_{r} p_{s} k} M \leq M, \quad \forall r, s \in R, \forall k \in K .
\end{aligned}
$$

Constraints (13) and (14) indicate that if two stations are successively served by a transfer vehicle, then the arrival time of demand in the latter station is equal to the sum of the arrival time of demand in the previous demand point and the travel time between the two demand points.

$$
a_{r}+c_{p_{r} j}-a_{s}+Y_{p_{r} p_{s} k} M \leq M, \quad \forall r \in R, \forall k \in K, \forall j \in D,
$$

$$
a_{s}-a_{r}-c_{p_{r} j}+Y_{p_{r} p_{s} k} M \leq M, \quad \forall r \in R, \forall k \in K, \forall j \in D .
$$

Constraints (15) and (16) indicate that the time for the passenger to reach the target station at the last demand station of the connecting vehicle is equal to the sum of the travel time of the vehicle to reach the demand point and the travel time between the two demand points.

$$
e_{r} \leq \sum_{k \in K} \sum_{t \in T} X_{r k t} T_{t}, \quad \forall r \in R
$$

Constraint (17) ensures that the vehicle arrives at the target station no later than the departure time of the target station.

\section{Model Solution}

The flexible bus route optimization scheduling problem for multiobjective stations is a typical NP-hard problem. As the problem scale expands, the computation likewise increases exponentially. Therefore, this kind of problem is usually solved by heuristic algorithm that can guarantee calculation speed and accuracy. Inspired by the law of universal gravitation, the present study proposes a heuristic algorithm that first generates the initial solution on the basis of the gravitation model. Then, the route is separately improved by the inter-route and intra-route optimization algorithm to obtain the final route. Figure 1 is the overall flow of the heuristic algorithm based on the gravity model and is the overall description of the following steps 1 to 4 .

\subsection{Passenger Travel Reservation and Demand Distribution.} First, the passenger travel reservation and demand allocation are completed according to the following steps.

Step 1: passengers make travel reservations according to their travel needs. Each passenger transmits his/her departure station, target station, expected arrival time, and other information to the travel reservation platform. Considering their dynamic changing characteristics, passenger demand on reservation platforms and travel time between connecting vehicle stations are updated every five minutes.

Step 2: travel reservation platform clusters all passengers according to the target station and expected arrival time of each passenger according to the principle that the actual arrival time is not later than the expected arrival time of passengers.

Step 3: results are generated according to the path in Sections 2.2 and 2.3. The arrival time of the transfer vehicle at each demand point is preliminarily estimated on the basis of several factors, including the expected arrival time of the transfer vehicle, average driving speed, position of the passenger demand point, and number of passengers at each demand point.

Step 4: the preliminarily estimated time of the connecting vehicle arriving at each demand point is sent to the passengers of the corresponding demand point. Passenger can choose whether to take the bus or wait for the connecting vehicle according to the rationality of its arrival time.

\subsection{Generating Initial Vehicle Path Solution on the Basis of the} Gravity Model. The path search problem is transformed into the iterative problem of the site selection chain, which is most attractive to the current site based on the gravity model algorithm. The gravity between the two sites is defined as follows.

$$
F_{i j}=\frac{N_{i} N_{j}}{c_{i j}^{2}},
$$

where $N_{i}$ is the number of passengers at station $i$ and $c_{i j}$ is the travel time between station $i$ and station $j$. The higher value of $F_{i j}$ means that the two stations have more passengers and less travel cost, thus needing priority service. Therefore, station $j$ should be set as the next station of station $I$.

Given the rated vehicle carrying capacity, the steps of the gravity model algorithm to generate the initial path solution are as follows.

Step 1: determine the vehicle starting point. Initial $k=1$. Randomly select one station with passenger boarding requirements as the starting point of vehicle $k$.

Step 2: determine if similar passengers are not serviced. If so, proceed to Step 3. Otherwise, proceed to Step 5.

Step 3: search for the next site. The most attractive station $X$ is that between the current station and that in the boarding station with similar passengers. Station $X$ is then added to the route selection chain, and the number of passengers is calculated after the vehicle arrives at the station, and the time to arrive at the target site after joining station $X$.

Step 4: determine whether the vehicle route is reasonable after joining station $X$. If the number of passengers served by the current vehicle does not exceed the on-board capacity $Q k$ and the time to reach the target station does not exceed the time required by passengers, then station $X$ shall be taken as a new starting point. Thus, Step 5 is skipped. Otherwise, proceed to Step 5 . 


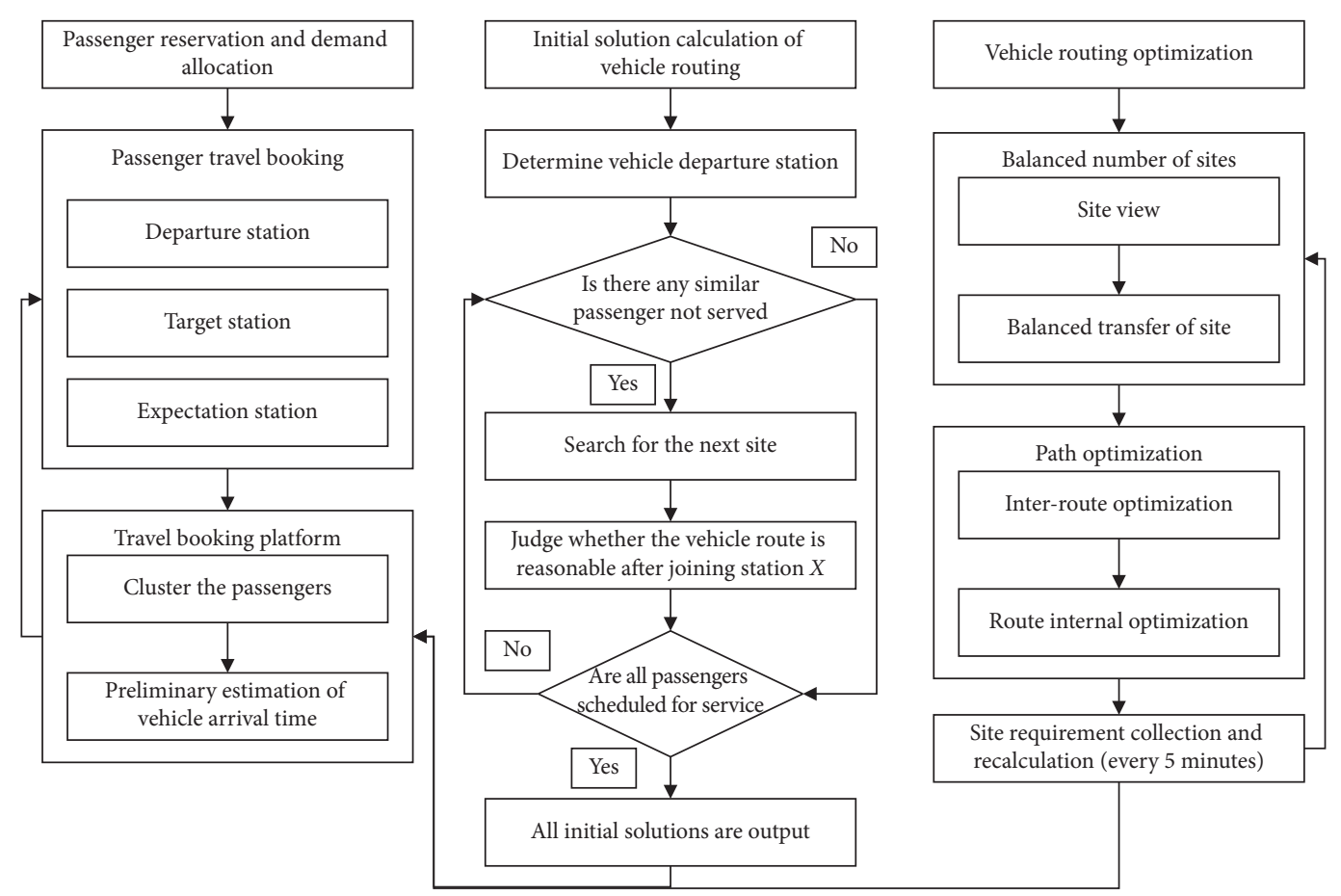

Figure 1: Flexible bus route optimization scheduling process responding to dynamic demand.

Step 5: determine if all classes of passengers are served. If several passengers are not scheduled, then the next car should be dispatched, $k=k+1$ and go back to Step 1. Otherwise, all current initial paths are output, and the initial vehicle path solution generation algorithm based on the gravity model is completed.

3.3. Vehicle Routing Optimization Based on Station Equilibrium and Exchange. This section introduces the route optimization algorithm between and within routes to further improve the route quality and passenger service level. Notably, Steps 1 and 2 of the algorithm belong to inter-vehicle path optimization. During their execution, multiple feasible route solutions may be searched. If only the current optimal set of solutions is saved during the search and Step 3 is performed, the final route result may not be optimal. Therefore, the proposed algorithm saves all feasible solution groups found in Steps 1and 2, and Step 3 is performed for each feasible solution group. Moreover, the objective function of all route solution groups is comprehensively evaluated so as to find the final optimal solution.

Step 1: first, the number of stations between the vehicles serving the target site and taking the same time to reach the target site is equalized. Service stations are checked for any imbalance in this number. If found, then on the premise of meeting the requirements of vehicle capacity and time to reach the target site, several vehicle routes that need to pass through more sites are transferred to the vehicle routes that need to pass through fewer sites. Thus, reasonable site sequence is arranged.

Step 2: the path between vehicles that serve the target site and those that need the same amount of time to reach the target site is optimized. The main application between the two routes is the exchange of two sites and search for better routes. In the process of exchange optimization, vehicle capacity and time to reach the target site are guaranteed.

Step 3: route of each car is internally optimized. The order of the two stops is exchanged mainly within the same vehicle route to evaluate whether the objective function value is reduced. If so, then site order is exchanged. Otherwise, this exchange is abandoned. After a certain number of attempts, the algorithm is terminated and the final route result is output.

Step 4: considering the dynamic variation characteristics of passenger demand and the variation characteristics of travel time between stations, the requirements of each station are collected and recalculated every five minutes, and Steps 1-3 above are repeated.

After the above four steps, the objective function of the route scheduling model can be optimized on the premise of ensuring that passengers arrive at the target station according to the expected time. The entire service time can be reduced and the route of each vehicle is more reasonable than before. In addition, the running cost of vehicles and waiting time of passengers can be reduced while service quality and efficiency can be improved.

\section{Case Analysis}

Huilongguan District of Beijing is a densely populated area of commuters, with large travel demand during peak hours, and the travel time of travelers with different work nature 
TABLE 2: Constant variables in the case.

\begin{tabular}{lccc}
\hline Variable & Variable name & Number of variables & Unit \\
\hline$H$ & Number of demand points & 15 & Unit \\
$D$ & Number of target stations & 3 & Unit \\
$Q$ & Vehicle rated passenger capacity & 7 & Person \\
$V$ & Fleet size/vehicle & 18 & Car \\
$W$ & Expected arrival time/minute & $30 / 40 / 50$ & Minute \\
$F$ & Passenger arrival and boarding time/minute & 0.5 & Minute \\
\hline
\end{tabular}

TABle 3: The quantity demanded at the initial moment.

\begin{tabular}{|c|c|c|c|}
\hline Passenger number & Departure station & Target station & Expected arrival time/minute \\
\hline \multicolumn{4}{|c|}{ (a) Passenger demand with target station 1} \\
\hline 1 & 1 & 1 & 30 \\
\hline 2 & 1 & 1 & 50 \\
\hline 3 & 1 & 1 & 50 \\
\hline 4 & 2 & 1 & 30 \\
\hline 5 & 2 & 1 & 50 \\
\hline 6 & 3 & 1 & 30 \\
\hline 7 & 3 & 1 & 40 \\
\hline 8 & 4 & 1 & 30 \\
\hline 9 & 5 & 1 & 30 \\
\hline 10 & 5 & 1 & 40 \\
\hline 11 & 5 & 1 & 50 \\
\hline 12 & 5 & 1 & 50 \\
\hline 13 & 6 & 1 & 30 \\
\hline 14 & 6 & 1 & 40 \\
\hline 15 & 7 & 1 & 30 \\
\hline 16 & 7 & 1 & 50 \\
\hline 17 & 7 & 1 & 50 \\
\hline 18 & 8 & 1 & 30 \\
\hline 19 & 8 & 1 & 40 \\
\hline 20 & 9 & 1 & 40 \\
\hline 21 & 10 & 1 & 40 \\
\hline 22 & 10 & 1 & 50 \\
\hline 23 & 11 & 1 & 40 \\
\hline 24 & 11 & 1 & 50 \\
\hline 25 & 12 & 1 & 30 \\
\hline 26 & 12 & 1 & 50 \\
\hline 27 & 13 & 1 & 30 \\
\hline 28 & 14 & 1 & 40 \\
\hline 29 & 14 & 1 & 40 \\
\hline 30 & 15 & 1 & 40 \\
\hline \multicolumn{4}{|c|}{ (b) Passenger demand with target station 2} \\
\hline 31 & 1 & 2 & 30 \\
\hline 32 & 1 & 2 & 50 \\
\hline 33 & 2 & 2 & 30 \\
\hline 34 & 2 & 2 & 50 \\
\hline 35 & 3 & 2 & 30 \\
\hline 36 & 3 & 2 & 40 \\
\hline 37 & 3 & 2 & 50 \\
\hline 38 & 4 & 2 & 30 \\
\hline 39 & 5 & 2 & 30 \\
\hline 40 & 5 & 2 & 40 \\
\hline 41 & 5 & 2 & 50 \\
\hline 42 & 6 & 2 & 30 \\
\hline 43 & 6 & 2 & 40 \\
\hline 44 & 6 & 2 & 50 \\
\hline 45 & 7 & 2 & 30 \\
\hline 46 & 7 & 2 & 50 \\
\hline
\end{tabular}


TABLE 3: Continued.

\begin{tabular}{|c|c|c|c|}
\hline Passenger number & Departure station & Target station & Expected arrival time/minute \\
\hline 47 & 8 & 2 & 30 \\
\hline 48 & 8 & 2 & 40 \\
\hline 49 & 8 & 2 & 50 \\
\hline 50 & 9 & 2 & 40 \\
\hline 51 & 10 & 2 & 40 \\
\hline 52 & 10 & 2 & 50 \\
\hline 53 & 11 & 2 & 40 \\
\hline 54 & 12 & 2 & 30 \\
\hline 55 & 12 & 2 & 50 \\
\hline 56 & 12 & 2 & 50 \\
\hline 57 & 13 & 2 & 30 \\
\hline 58 & 14 & 2 & 40 \\
\hline 59 & 15 & 2 & 40 \\
\hline 60 & 15 & 2 & 40 \\
\hline \multicolumn{4}{|c|}{ (c) Passenger demand with target station 3} \\
\hline 61 & 1 & 3 & 30 \\
\hline 62 & 1 & 3 & 50 \\
\hline 63 & 1 & 3 & 50 \\
\hline 64 & 2 & 3 & 30 \\
\hline 65 & 2 & 3 & 50 \\
\hline 66 & 3 & 3 & 30 \\
\hline 67 & 3 & 3 & 40 \\
\hline 68 & 4 & 3 & 30 \\
\hline 69 & 5 & 3 & 30 \\
\hline 70 & 5 & 3 & 40 \\
\hline 71 & 5 & 3 & 50 \\
\hline 72 & 5 & 3 & 50 \\
\hline 73 & 6 & 3 & 30 \\
\hline 74 & 6 & 3 & 40 \\
\hline 75 & 7 & 3 & 30 \\
\hline 76 & 7 & 3 & 50 \\
\hline 77 & 8 & 3 & 30 \\
\hline 78 & 8 & 3 & 40 \\
\hline 79 & 8 & 3 & 50 \\
\hline 80 & 9 & 3 & 40 \\
\hline 21 & 10 & 1 & 40 \\
\hline 22 & 10 & 1 & 50 \\
\hline 23 & 11 & 1 & 40 \\
\hline 24 & 11 & 1 & 50 \\
\hline 25 & 12 & 1 & 30 \\
\hline 26 & 12 & 1 & 50 \\
\hline 27 & 13 & 1 & 30 \\
\hline 28 & 14 & 1 & 40 \\
\hline 29 & 14 & 1 & 40 \\
\hline 30 & 15 & 1 & 40 \\
\hline
\end{tabular}

and commuting distance varies greatly. Therefore, it is suitable to be used as a case study of demand responsive flexible public transport model. In order to facilitate the model analysis, this paper abstracts the Huilongguan regional public transport network and retains the network topology.

A small network is used as case solution to verify the accuracy and applicability of the above model. Table 2 shows the input parameter of the constant variable of a small network, whereas Table 3 shows the passenger demand of each station at the initial moment. Table 4 shows the travel time matrix between stations at the initial time, where $H$ is the demand point and $D$ is the target station.

Using the heuristic algorithm proposed in this paper to solve the numerical case of demand at the initial moment obtains the service path of each vehicle, number of passengers served, and objective function corresponding to each path, as shown in Table 5.

Then, with the dynamic change of passenger demand and travel time between stations, the above path planning 
TABLE 4: Case network travel time matrix at the initial moment.

\begin{tabular}{|c|c|c|c|c|c|c|c|c|c|c|c|c|c|c|c|c|c|c|}
\hline & $H 1$ & $H 2$ & H3 & $H 4$ & H5 & H6 & $H 7$ & H8 & H9 & $H 10$ & $H 11$ & $H 12$ & $H 13$ & H14 & H15 & $D 1$ & D2 & D3 \\
\hline$H 1$ & 0 & 3 & 6 & 5 & 4 & 5 & 8 & 6 & 9 & 9 & 6 & 3 & 3 & 4 & 8 & 3 & 9 & 9 \\
\hline$H 2$ & 7 & 0 & 6 & 9 & 8 & 3 & 5 & 5 & 4 & 7 & 9 & 3 & 3 & 3 & 6 & 3 & 8 & 7 \\
\hline H3 & 9 & 6 & 0 & 6 & 5 & 8 & 8 & 8 & 3 & 7 & 9 & 4 & 5 & 5 & 5 & 9 & 7 & 9 \\
\hline H4 & 4 & 7 & 6 & 0 & 9 & 7 & 8 & 5 & 5 & 7 & 7 & 4 & 5 & 6 & 8 & 9 & 5 & 6 \\
\hline H5 & 5 & 3 & 8 & 8 & 0 & 5 & 7 & 9 & 5 & 6 & 3 & 8 & 6 & 5 & 8 & 9 & 6 & 5 \\
\hline H6 & 8 & 3 & 5 & 9 & 5 & 0 & 5 & 4 & 5 & 9 & 9 & 8 & 7 & 8 & 6 & 9 & 8 & 3 \\
\hline$H 7$ & 9 & 8 & 4 & 5 & 6 & 8 & 0 & 3 & 4 & 8 & 6 & 8 & 8 & 8 & 5 & 6 & 7 & 6 \\
\hline H8 & 5 & 5 & 5 & 7 & 5 & 6 & 6 & 0 & 6 & 4 & 4 & 9 & 5 & 8 & 5 & 8 & 5 & 6 \\
\hline H9 & 3 & 5 & 8 & 5 & 4 & 5 & 5 & 6 & 0 & 7 & 3 & 6 & 8 & 3 & 3 & 6 & 8 & 3 \\
\hline$H 10$ & 5 & 9 & 9 & 5 & 9 & 4 & 5 & 5 & 5 & 0 & 9 & 9 & 4 & 3 & 5 & 6 & 8 & 7 \\
\hline$H 11$ & 5 & 3 & 7 & 6 & 6 & 7 & 8 & 3 & 3 & 4 & 0 & 7 & 4 & 9 & 6 & 3 & 7 & 9 \\
\hline$H 12$ & 5 & 3 & 9 & 9 & 6 & 6 & 7 & 7 & 9 & 5 & 9 & 0 & 7 & 3 & 5 & 5 & 5 & 4 \\
\hline$H 13$ & 7 & 5 & 7 & 9 & 7 & 8 & 7 & 5 & 3 & 7 & 5 & 7 & 0 & 5 & 5 & 6 & 5 & 5 \\
\hline$H 14$ & 9 & 5 & 6 & 5 & 6 & 4 & 8 & 5 & 8 & 9 & 5 & 9 & 9 & 0 & 6 & 8 & 8 & 9 \\
\hline$H 15$ & 6 & 9 & 6 & 4 & 8 & 8 & 3 & 4 & 5 & 3 & 4 & 5 & 9 & 4 & 0 & 7 & 9 & 6 \\
\hline
\end{tabular}

TABLE 5: Vehicle service path results for the initial moment.

\begin{tabular}{lcccc}
\hline $\begin{array}{l}\text { Passenger } \\
\text { number }\end{array}$ & Vehicle path & $\begin{array}{c}\text { Number of passengers } \\
\text { served }\end{array}$ & $\begin{array}{c}\text { Expected arrival time/ } \\
\text { minute }\end{array}$ & $\begin{array}{c}\text { The target function value corresponding to } \\
\text { the path }\end{array}$ \\
\hline 1 & $5-2-3-12-4-D_{1}$ & $1-1-1-1-1$ & 30 & 83 \\
2 & $13-1-7-8-6-D_{1}$ & $1-1-1-1-1$ & 30 & 92 \\
3 & $14-3-11-15-10-$ & $2-1-1-1-1$ & 40 & 123 \\
4 & $D_{1}$ & $1-1-1-1$ & 40 & 109 \\
5 & $8-5-6-9-D_{1}$ & $2-1-1$ & 50 & 156 \\
6 & $1-2-10-D_{1}$ & $2-1-1-2$ & 50 & 216 \\
7 & $7-11-12-5-D_{1}$ & $1-1-1-1-1-1$ & 30 & 102 \\
8 & $5-13-6-3-12-7-$ & $1-1-1-1$ & 30 & 72 \\
9 & $D_{2}$ & $1-2-1-1-1$ & 40 & 130 \\
10 & $4-1-8-2-D_{2}$ & $1-1-1-1$ & 40 & 111 \\
11 & $6-15-3-9-11-D_{2}$ & $1-1-1-1-1$ & 50 & 163 \\
12 & $8-14-10-5-D_{2}$ & $2-1-1-1$ & 50 & 180 \\
13 & $7-2-6-1-3-D_{2}$ & $1-1-1-1-1$ & 30 & 85 \\
14 & $12-8-10-5-D_{2}$ & $1-1-1-1-1$ & 30 & 86 \\
15 & $5-2-12-7-3-D_{3}$ & $2-1-1-1-1$ & 40 & 135 \\
16 & $4-8-6-1-13-D_{3}$ & $1-1-1-1$ & 40 & 113 \\
17 & $15-10-6-9-14-$ & $1-1-1$ & 50 & 125 \\
18 & $3-8-11-5-D_{3}$ & $8-10-2-D_{3}$ & 50 & 235
\end{tabular}

Note. The vehicle path in the table above is represented by the number sequence of the passenger demand points passed by. For example, 5-2-3-12-4-D1 indicates that the vehicle passes through the passenger demand points numbered 5,2,3,12, and 4 in turn and finally arrives at the target station numbered $D 1$. "Number of passengers served" corresponds to "vehicle path." For example, in the data in row 5, "vehicle path" is 1-2-10-D1, and number of passengers served is 2-1-1, which means that the number of passengers served at the passenger demand point with No. 1 is 2 , and the number of passengers served at the passenger demand point Nos. 2 and 10 is 1.

results do not meet the current passenger demand and real situation. Thus, new path planning was needed. At this point, the reservation platform updates the travel time between the new passenger demand and the connecting vehicle station after the first five minutes of the summary. Table 6 shows the quantity demanded within the first five minutes after the initial moment. Table 7 shows the case network travel time matrix at the first five minutes. Table 8 shows the vehicle service path results for the first five minutes.

Then, such platforms are solved again using the method described in Section 2.2. Figure 2 shows the schematic diagram of vehicle routing results.

According to the calculation results in Table 8, Figure 2 shows the network topology of some vehicle driving paths. As Table 8 has a lot of contents, three groups of routes are 
TABLE 6: The quantity demanded within the first 5 minutes.

\begin{tabular}{|c|c|c|c|}
\hline Passenger number & Departure station & Target station & Expected arrival time/minute \\
\hline \multicolumn{4}{|c|}{ (a) Passenger demand with target station 1} \\
\hline 1 & 1 & 1 & 30 \\
\hline 2 & 1 & 1 & 50 \\
\hline 3 & 1 & 1 & 50 \\
\hline 4 & 2 & 1 & 30 \\
\hline 5 & 2 & 1 & 50 \\
\hline 6 & 3 & 1 & 30 \\
\hline 7 & 3 & 1 & 40 \\
\hline 8 & 4 & 1 & 30 \\
\hline 9 & 5 & 1 & 30 \\
\hline 10 & 5 & 1 & 30 \\
\hline 11 & 5 & 1 & 30 \\
\hline 12 & 5 & 1 & 30 \\
\hline 13 & 5 & 1 & 40 \\
\hline 14 & 5 & 1 & 50 \\
\hline 15 & 5 & 1 & 50 \\
\hline 16 & 6 & 1 & 30 \\
\hline 17 & 6 & 1 & 40 \\
\hline 18 & 7 & 1 & 30 \\
\hline 19 & 7 & 1 & 50 \\
\hline 20 & 7 & 1 & 50 \\
\hline 21 & 8 & 1 & 30 \\
\hline 22 & 8 & 1 & 40 \\
\hline 23 & 9 & 1 & 40 \\
\hline 24 & 10 & 1 & 40 \\
\hline 25 & 10 & 1 & 50 \\
\hline 26 & 11 & 1 & 40 \\
\hline 27 & 11 & 1 & 50 \\
\hline 28 & 12 & 1 & 30 \\
\hline 29 & 12 & 1 & 50 \\
\hline 30 & 13 & 1 & 30 \\
\hline 31 & 14 & 1 & 40 \\
\hline 32 & 14 & 1 & 40 \\
\hline 33 & 15 & 1 & 40 \\
\hline 34 & 15 & 1 & 50 \\
\hline \multicolumn{4}{|c|}{ (b) Passenger demand with target station 2} \\
\hline 35 & 1 & 2 & 30 \\
\hline 36 & 1 & 2 & 50 \\
\hline 37 & 2 & 2 & 30 \\
\hline 38 & 2 & 2 & 50 \\
\hline 39 & 2 & 2 & 50 \\
\hline 40 & 3 & 2 & 30 \\
\hline 41 & 3 & 2 & 40 \\
\hline 42 & 3 & 2 & 50 \\
\hline 43 & 4 & 2 & 30 \\
\hline 44 & 5 & 2 & 30 \\
\hline 45 & 5 & 2 & 40 \\
\hline 46 & 5 & 2 & 50 \\
\hline 47 & 6 & 2 & 30 \\
\hline 48 & 6 & 2 & 40 \\
\hline 49 & 6 & 2 & 50 \\
\hline 50 & 7 & 2 & 30 \\
\hline 51 & 7 & 2 & 50 \\
\hline 52 & 8 & 2 & 30 \\
\hline 53 & 8 & 2 & 40 \\
\hline 54 & 8 & 2 & 50 \\
\hline 55 & 9 & 2 & 40 \\
\hline 56 & 10 & 2 & 40 \\
\hline 57 & 10 & 2 & 40 \\
\hline 58 & 10 & 2 & 50 \\
\hline
\end{tabular}


TABLE 6: Continued.

\begin{tabular}{|c|c|c|c|}
\hline Passenger number & Departure station & Target station & Expected arrival time/minute \\
\hline 59 & 11 & 2 & 40 \\
\hline 60 & 12 & 2 & 30 \\
\hline 61 & 12 & 2 & 50 \\
\hline 62 & 12 & 2 & 50 \\
\hline 63 & 13 & 2 & 30 \\
\hline 64 & 14 & 2 & 40 \\
\hline 65 & 15 & 2 & 40 \\
\hline 66 & 15 & 2 & 40 \\
\hline 67 & 1 & 3 & 30 \\
\hline 68 & 1 & 3 & 30 \\
\hline \multicolumn{4}{|c|}{ (c) Passenger demand with target station 3} \\
\hline 69 & 1 & 3 & 50 \\
\hline 70 & 1 & 3 & 50 \\
\hline 71 & 2 & 3 & 30 \\
\hline 72 & 2 & 3 & 40 \\
\hline 73 & 2 & 3 & 50 \\
\hline 74 & 3 & 3 & 30 \\
\hline 75 & 3 & 3 & 40 \\
\hline 76 & 3 & 3 & 50 \\
\hline 77 & 4 & 3 & 30 \\
\hline 78 & 5 & 3 & 30 \\
\hline 79 & 5 & 3 & 40 \\
\hline 80 & 5 & 3 & 50 \\
\hline 81 & 5 & 3 & 50 \\
\hline 82 & 6 & 3 & 30 \\
\hline 83 & 6 & 3 & 40 \\
\hline 84 & 7 & 3 & 30 \\
\hline 85 & 7 & 3 & 50 \\
\hline 86 & 8 & 3 & 30 \\
\hline 87 & 8 & 3 & 40 \\
\hline 88 & 8 & 3 & 50 \\
\hline 89 & 9 & 3 & 40 \\
\hline 90 & 10 & 3 & 40 \\
\hline 91 & 10 & 3 & 50 \\
\hline 92 & 11 & 3 & 40 \\
\hline 93 & 12 & 3 & 30 \\
\hline 94 & 12 & 3 & 50 \\
\hline 95 & 12 & 3 & 50 \\
\hline 96 & 13 & 3 & 30 \\
\hline 97 & 13 & 3 & 30 \\
\hline 98 & 14 & 3 & 40 \\
\hline 99 & 15 & 3 & 40 \\
\hline 100 & 15 & 3 & 40 \\
\hline 101 & 15 & 3 & 40 \\
\hline 102 & 15 & 3 & 40 \\
\hline
\end{tabular}

selected and displayed according to the passenger destination; with $D_{1}$ as the destination, we show the path 5-2-3-124- $D_{1}$; with $D_{2}$ as the destination, we show the paths 5-13-6-312-7- $D_{2}$; with $D_{3}$ as the destination, we show the path 8-10$2-D_{3}$.

A total of 102 travel demands of 15 randomly generated demand points in 100 groups are tested by the model. The objective is to further verify the algorithm's reliability, with the existing constant variables and the travel time matrix table of the case network unchanged. Figures 3-5 show the test results. Under the given conditions, all the 102 travel demands are completed, the number of vehicles required is $17-21$, and the total travel time of $84 \%$ vehicles is $552-624$ minutes (as shown in Figure 3). Moreover, the average travel time of each vehicle is 24.59 minutes, indicating that the travel time of vehicles solved by the heuristic algorithm is 
TABLE 7: Case network travel time matrix at the first 5 minutes.

\begin{tabular}{ccccccccccccccccccc}
\hline & $H_{1}$ & $H_{2}$ & $H_{3}$ & $H_{4}$ & $H_{5}$ & $H_{6}$ & $H_{7}$ & $H_{8}$ & $H_{9}$ & $H_{10}$ & $H_{11}$ & $H_{12}$ & $H_{13}$ & $H_{14}$ & $H_{15}$ & $D_{1}$ & $D_{2}$ & $D_{3}$ \\
\hline$H_{1}$ & 0 & 7 & 8 & 9 & 5 & 7 & 7 & 4 & 9 & 6 & 4 & 3 & 7 & 6 & 5 & 5 & 3 & 3 \\
$H_{2}$ & 6 & 0 & 3 & 5 & 4 & 9 & 7 & 9 & 3 & 4 & 4 & 3 & 8 & 3 & 7 & 9 & 7 & 9 \\
$H_{3}$ & 6 & 8 & 0 & 7 & 5 & 4 & 3 & 5 & 8 & 5 & 4 & 8 & 3 & 7 & 8 & 6 & 5 & 3 \\
$H_{4}$ & 9 & 5 & 7 & 0 & 9 & 3 & 6 & 5 & 5 & 6 & 3 & 8 & 8 & 5 & 5 & 8 & 9 & 4 \\
$H_{5}$ & 8 & 5 & 8 & 8 & 0 & 6 & 9 & 8 & 9 & 7 & 3 & 3 & 7 & 4 & 6 & 6 & 4 & 3 \\
$H_{6}$ & 5 & 3 & 4 & 9 & 5 & 0 & 5 & 6 & 6 & 4 & 9 & 5 & 7 & 8 & 9 & 8 & 4 & 7 \\
$H_{7}$ & 7 & 8 & 4 & 5 & 7 & 8 & 0 & 9 & 5 & 7 & 7 & 8 & 7 & 4 & 5 & 8 & 8 & 3 \\
$H_{8}$ & 7 & 5 & 6 & 5 & 5 & 5 & 8 & 0 & 3 & 6 & 5 & 4 & 6 & 6 & 5 & 4 & 5 & 8 \\
$H_{9}$ & 9 & 6 & 5 & 7 & 8 & 8 & 3 & 4 & 0 & 7 & 3 & 4 & 7 & 4 & 5 & 3 & 3 & 3 \\
$H_{10}$ & 9 & 3 & 9 & 5 & 8 & 6 & 5 & 3 & 6 & 0 & 7 & 4 & 6 & 9 & 8 & 6 & 8 & 6 \\
$H_{11}$ & 9 & 8 & 4 & 3 & 9 & 9 & 6 & 5 & 7 & 5 & 0 & 3 & 3 & 7 & 3 & 9 & 5 & 7 \\
$H_{12}$ & 7 & 3 & 3 & 4 & 9 & 4 & 9 & 9 & 7 & 6 & 3 & 0 & 3 & 9 & 9 & 3 & 9 & 9 \\
$H_{13}$ & 7 & 3 & 5 & 6 & 7 & 4 & 7 & 8 & 3 & 8 & 7 & 8 & 0 & 3 & 7 & 6 & 3 & 5 \\
$H_{14}$ & 4 & 8 & 6 & 5 & 7 & 7 & 6 & 8 & 5 & 9 & 7 & 8 & 9 & 0 & 4 & 6 & 5 & 8 \\
$H_{15}$ & 6 & 3 & 9 & 4 & 8 & 4 & 9 & 6 & 7 & 6 & 3 & 3 & 9 & 6 & 0 & 7 & 6 & 5 \\
\hline
\end{tabular}

TABLE 8: Vehicle service path results for the first 5 minutes.

\begin{tabular}{lcccc}
\hline $\begin{array}{l}\text { Passenger } \\
\text { number }\end{array}$ & Vehicle path & $\begin{array}{c}\text { Number of passengers } \\
\text { served }\end{array}$ & $\begin{array}{c}\text { Expected arrival time/ } \\
\text { minute }\end{array}$ & $\begin{array}{c}\text { The target function value corresponding to } \\
\text { the path }\end{array}$ \\
\hline 1 & $5-2-3-12-4-D_{1}$ & $2-1-1-1-1$ & 30 & 84 \\
2 & $13-1-7-8-6-5-D_{1}$ & $1-1-1-1-1-2$ & 30 & 132 \\
3 & $14-3-11-15-10-D_{1}$ & $2-1-1-1-1$ & 40 & 123 \\
4 & $8-5-6-9-D_{1}$ & $1-1-1-1$ & 40 & 109 \\
5 & $1-2-10-D_{1}$ & $2-1-1$ & 50 & 156 \\
6 & $7-11-15-12-5-D_{1}$ & $2-1-1-1-2$ & 50 & 259 \\
7 & $5-13-6-3-12-7-D_{2}$ & $1-1-1-1-1-1$ & 30 & 102 \\
8 & $4-1-8-2-D_{2}$ & $1-1-1-1$ & 30 & 72 \\
9 & $6-15-3-9-11-D_{2}$ & $1-2-1-1-1$ & 40 & 130 \\
10 & $8-14-10-5-10-D_{2}$ & $1-1-1-1-1$ & 40 & 131 \\
11 & $7-2-6-1-3-D_{2}$ & $1-1-1-1-1$ & 50 & 163 \\
12 & $12-8-10-5-2-D_{2}$ & $2-1-1-1-1$ & 50 & 191 \\
13 & $5-2-12-7-3-D_{3}$ & $1-1-1-1-1$ & 30 & 85 \\
14 & $4-8-6-1-13-1-D_{3}$ & $1-1-1-1-1-1$ & 30 & 95 \\
15 & $15-10-6-9-14-D_{3}$ & $2-1-1-1-1$ & 40 & 135 \\
16 & $3-8-15-11-5-13-$ & $1-1-2-1-1-1-1$ & 40 & 175 \\
17 & $2-D_{3}$ & $1-1-1$ & 50 & 125 \\
18 & $8-10-2-D_{3}$ & $2-2-1-2-1$ & 50 & 288 \\
\hline
\end{tabular}

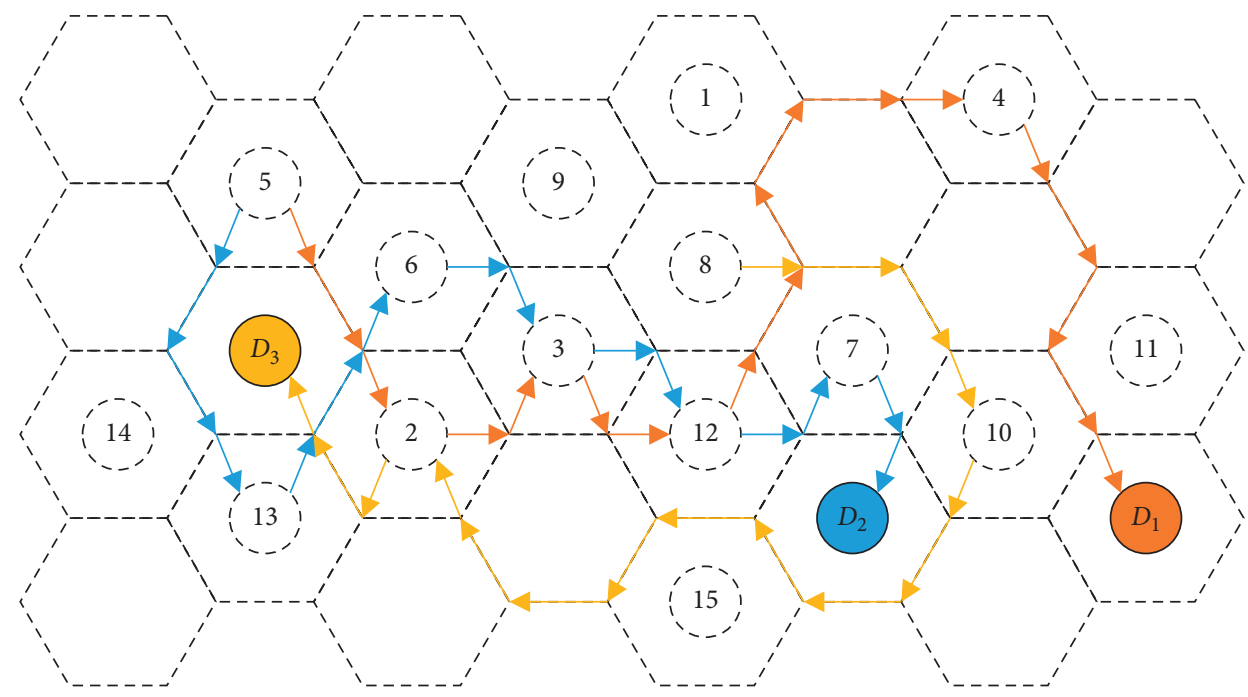

Passenger target station
Destination is the path of $D_{1}$ Destination is the path of $D_{3}$

Figure 2: The schematic diagram of vehicle routing results. 


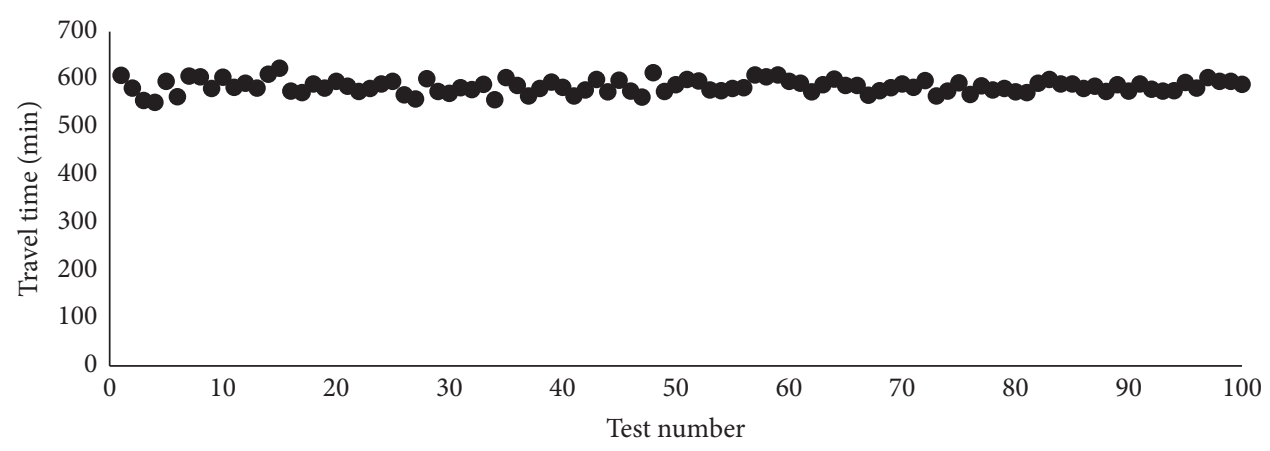

FIGURE 3: Vehicle routing results.

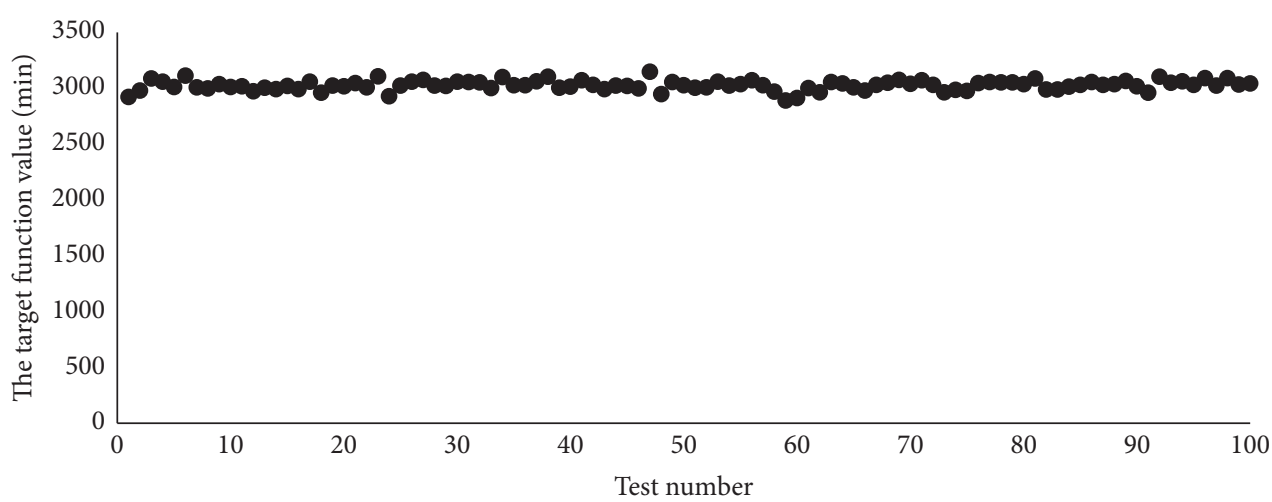

FIgURE 4: Total objective function result distribution.

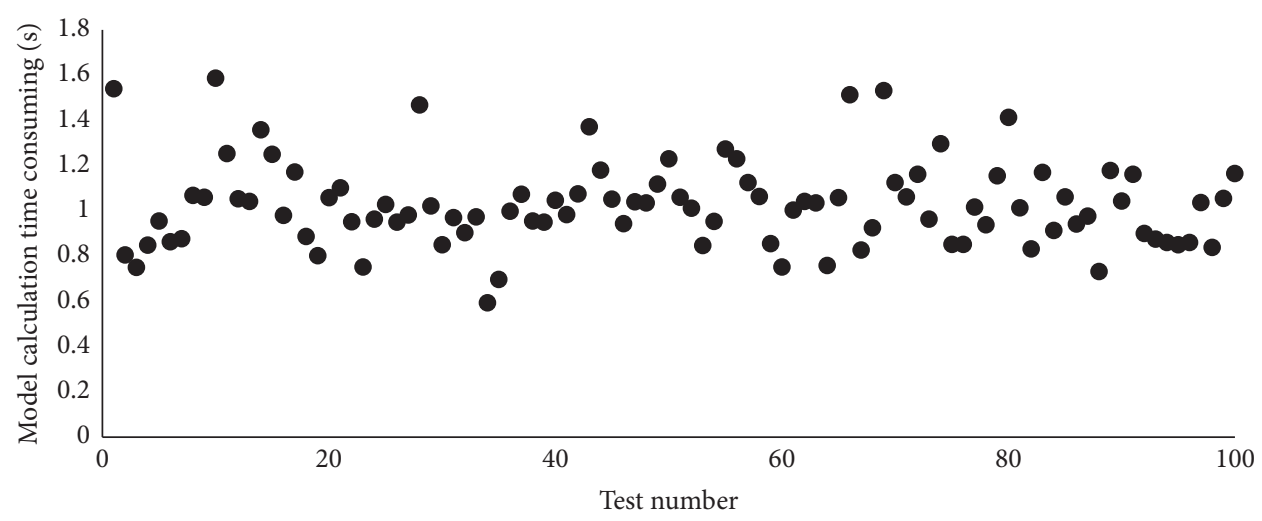

FIGURE 5: The time distribution of the model is calculated.

relatively stable. Although the demand distribution is highly uncertain, stable and reliable results are still generated due to the high robustness of the model and algorithm. The total objective function value remains mostly concentrated between 2888 and 3146 under the influence of demand changes (as shown in Figure 4). At the same time, the solution time of all 100 groups of data is less than 1.6 seconds, and the calculation time is 1.03 seconds on average (as shown in Figure 5). Thus, the algorithm proposed in this study is applicable to the actual system and the vehicle path optimization and collaborative scheduling can be completed within the limited time window of reservation and service provision.

\section{Conclusion}

This study investigates the flexible bus route optimization scheduling problem. The proposed model is dynamically constructed on the basis of passenger needs and "many-tomany" flexible bus system (i.e., multiple flexible stations corresponding to multiple target sites). Moreover, the routes are considered according to the dynamic changes of passenger demand and real-time updated shuttle travel time. The routes are established to maximize passenger services, minimize travel time for all passengers, and minimize operating time for all vehicles. The example shows that the flexible bus route optimization scheduling model in 
response to dynamic demand can give more reasonable route optimization scheme and optimize passenger travel and vehicle operation cost compared with the model which ignores the change of passenger demand and takes the connection travel time as a fixed value.

In addition, we select more parameters in modeling and consider more factors, such as passenger demand, operating cost, etc., to make our solution to be nearer to the reality of the flexible bus system. At the same time, we focus on the actual travel demand for flexible bus scheduling in Huilongguan District of Beijing and establish the flexible bus scheduling model for multiple flexible stations and multiple target sites, which has more practicability than the "manyto-one" (i.e., multiple flexible stations corresponding to one target site) scheduling model.

For future research, we will select more areas to use our proposed flexible bus route optimization scheduling method, and then we will continually evaluate the effectiveness of the model for urban public transport operation and management and optimize the model and parameters.

\section{Data Availability}

The data used to support the findings of this study are available from the corresponding author upon request, and the reader can use MATLAB for verification.

\section{Conflicts of Interest}

The authors declare that they have no conflicts of interest.

\section{References}

[1] M. Flusberg, "An innovative public transportation system for a small city: the Merrill, Wisconsin, case study," Transportation Research Record, vol. 606, pp. 54-59, 1976.

[2] Y. Lei, P.-q. Lin, and K.-b. Yao, "The network scheduling model and its solution algorithm of internet customized shuttle bus," Journal of Transportation Systems Engineering and Information Technology, vol. 17, no. 1, pp. 157-163, 2017.

[3] L. Yun, Y.-s. Jiang, and X.-m. Song, "Introduction of mobility allowance shuttle transit system (MAST) and the summary of its research progress," Journal of Transportation Engineering and Information, vol. 7, no. 4, pp. 79-83, 2009.

[4] Y. Shen and H.-f. Guan, "Study on demand responsive transport system and implementation issues," Traffic \& Transportation, vol. 12, pp. 75-78, 2010.

[5] C. Peng and N. Yu, "Optimal design of demand adaptive paired-line hybrid transit: case of radial route structure," Transporation Research Part E: Logistics \& Transportation Review, vol. 110, pp. 71-89, 2018.

[6] G. Chen, Locating Flexible Feeder Bus Stations to Connect Urban Railway Systems, Shandong University, Shandong, China, 2015.

[7] C. Avishai, "Integrated smart feeder/shuttle transit service: simulation of new routing strategies," Journal of Advanced Transportation, vol. 47, pp. 595-618, 2013.

[8] S.-1 Pan, J. Yu, X.-1 Lu, and N. Zou, "Review of flexible transit service," Urban Transport of China, vol. 2, pp. 62-68, 2014.

[9] A. Pratelli and F. Schoen, "A mathematical programming model for the bus deviation route problem," Journal of the Operational Research Society, vol. 52, no. 5, pp. 494-502, 2001.
[10] Y.-w Lei, P.-q Lin, and K.-b Yao, "The network scheduling model and its solution algorithm of internet customized shuttle bus," Journal of Transportation Systems Engineering and Information Technology, vol. 17, no. 1, pp. 157-163, 2017.

[11] B. Vincent, J. I.-R Omar, and Á. R.-S Yasmin, "Vehicle and Crew scheduling for flexible bus transportation systems," Transportation Research Part B: Methodological, vol. 112, pp. 216-229, 2018.

[12] A. Giorgio, D. N. John, and B. Marco, "Enabling intermodal urban transport through complementary services: from flexible mobility services to the shared use mobility agency: workshop 4. developing intermodal transport systems," Research in Transportation Economics, vol. 59, pp. 179-184, 2016.

[13] S. M. Nourbakhsh and Y. Ouyang, "A structured flexible transit system for low demand areas," Transportation Research Part B: Methodological, vol. 1, no. 46, pp. 204-216, 2012.

[14] C. Mulley and D. N John, "Flexible transport services: a new market opportunity for public transport," Research in Transportation Economics, vol. 1, no. 25, pp. 39-45, 2009.

[15] B. Jenny and D. N. John, "A case study of flexible solutions to transport demand in a deregulated environment," Journal of Transport Geography, vol. 4, no. 15, pp. 262-273, 2007.

[16] L. Quadrifoglio and M. Dessouky, "Insertion heuristic for scheduling mobility allowance shuttle transit (MAST) services: sensitivity to service area," Computer-Aided Systems in Public Transport, Springer Series: Lecture Notes in Economics and Mathematical Systems, Berlin, Germany, 2007.

[17] L. Quadrifoglio and M. Dessouky, "Sensitivity analyses over the service area for mobility allowance shuttle transit (MAST) services," Computer-Aided Systems in Public Transport, pp. 419-432, Springer Series: Lecture Notes in Economics and Mathematical Systems, Berlin, Germany, 2008.

[18] X. Fu, Y. Gu, and Z. Liu, "Scheduling activity and travel patterns in multi-modal transit networks with customized bus services," Journal of Transportation Systems Engineering and Information Technology, vol. 19, no. 4, pp. 20-27, 2019.

[19] D. Koffman, Operational Experiences with Flexible Transit Services: A Synthesis of Transit Practice, Transportation Research Board, Washington DC, USA, 2015.

[20] K. Tsubouchi, "Adapted scheduling algorithm for on-demand bus system," International Journal of Intelligent Transportation Systems Research, vol. 10, no. 2, pp. 82-92, 2013.

[21] J. Xiong, Optimal Microcirculation System of Community Shuttle Linked with Metro Service, Beijing Jiaotong University, Beijing, China, 2015.

[22] Q.-f. Li, P. Chen, and Y. Nie, "Finding optimal hyperpaths in large transit networks with realistic headway distributions," European Journal of Operational Research, vol. 240, no. 1, pp. 98-108, 2015.

[23] P. Chen and Y. Nie, "Optimal transit routing with partial online information," Transportation Research Part B: Methodological, vol. 72, pp. 40-58, 2015.

[24] S. Pan, The Design Theory and Methodologdy of Flesaxible Microcirculation Transit System Oriented on the Transfer Station, Shandong University, Shandong, China, 2015.

[25] X. Guo, Research of Real-Time Custom Bus System Based on Demand Response, Beijing Jiaotong University, Beijing, China, 2016. 